\title{
6.25 Gb/s POF Link Using GaN $\mu$ LED Arrays and Optically Generated Pulse Amplitude Modulation
}

\author{
X. Li ${ }^{a}$, N. Bamiedakis ${ }^{a}$, J.L. Wei ${ }^{a}$, J. J. D. McKendry ${ }^{b}$, E. Xie ${ }^{b}$, R. Ferreira ${ }^{b}$, E. Gu ${ }^{\text {, }}$, \\ M. D. Dawson ${ }^{\text {, }}$ R. V. Penty ${ }^{\text {a }}$, I. H. White ${ }^{\mathrm{a}}$ \\ ${ }^{a}$ Electrical Engineering Division, Engineering Department, University of Cambridge, Cambridge CB3 OFA, UK, \\ ${ }^{b}$ Institute of Photonics, Department of Physics, SUPA, University of Strathclyde, Glasgow, G4 ONW, UK \\ E-mail address: xl336@cam.ac.uk
}

\begin{abstract}
Optically-generated PAM schemes using $\mu$ LED arrays are implemented for high-speed POF links for the first time. $6.25 \mathrm{~Gb} / \mathrm{s}$ PAM-16 transmission is demonstrated using $4 \mu \mathrm{LEDs}$, exhibiting $3.8 \mathrm{~dB}$ greater power-margin than a link with a single $\mu \mathrm{LED}$.

OCIS codes: (060.0060) Fiber optics and optical communications; (060.4080) Modulation
\end{abstract}

\section{Introduction}

Recently, data transmission using visible light-emitting-diodes (LEDs) over plastic optical fiber (POF) has drawn attention for ultra low-cost high-speed short-reach communication links, such as in-home networks and automotive information systems [1]. Unidirectional $5 \mathrm{~Gb} / \mathrm{s}$ data transmission over POF has been demonstrated using a single RCLED and an electrically-generated 32-level pulse amplitude modulation (PAM-32) modulation scheme [2]. However, the non-linear optical-power-versus-current characteristic of common LEDs introduces distortion and limits the optical system performance for advanced modulation formats [3]. Optical domain digital-to-analogue conversion (DAC) techniques have already been proposed for use in free-space LED-based links in order to mitigate the LED non-linearity [4, 5]. PAM, orthogonal frequency division multiplexing and multilevel pulse position modulation have been investigated using this method [5-7]. Moreover, optically-generated multilevel modulation has been implemented over multimode fibre links using 2-D vertical-cavity surface-emitting lasers [8].

In this paper therefore, we investigate the potential to achieve higher data transmission capacity in ultra lowcost POF links using optically-generated multilevel modulation schemes. This modelling and experimental study focuses on a POF link using micro-pixelated LED ( $\mu$ LED) arrays to optically generate a PAM modulation scheme. $\mu$ LEDs, with diameters $\leq 100 \mu \mathrm{m}$, are of great interest for use in low-cost optical links as they exhibit higher modulation bandwidths than conventional large-size LEDs [9]. Moreover, $\mu$ LEDs can be easily formed into arrays which achieve high coupling efficiency with POF owing to their small dimensions. Simulation results show that a greater system margin is available for optically-generated PAM links compared with electrically-generated PAM links. An optical link is set up over POF implementing an optically generated PAM-16 scheme using $4 \mu$ LEDs. Record $6.25 \mathrm{~Gb} / \mathrm{s}$ transmission with a bit-error-rate (BER) $<10^{-3}$ is achieved over $10 \mathrm{~m}$ of POF.

\section{Optically-generated PAM and simulation results}

For optically-generated PAM schemes, $N \mu$ LEDs are required in order to implement a $2^{\mathrm{N}}$-PAM scheme. Each $\mu$ LED is driven using on-off keying and the output power of the $\mu$ LEDs are adjusted to follow a $2^{\mathrm{N}}$ relationship. For each symbol, different combinations of $\mu$ LEDs are turned on to generate the corresponding signal levels. $4 \mu$ LEDs with an output power ratio of 1:2:4:8 are required to generate a PAM-16 signal [Fig. 1(a)]. The driving signal for each $\mu \mathrm{LED}$ can be generated by converting the input serial data sequence to 4 parallel data streams [Fig. 1(b)].

Basic modelling studies are carried out for this scheme using the link model presented in [2] and parameter components that match the actual devices using in the link demonstration. The obtained power budget for a POF link implemented with a single $\mu$ LED (electrically-generated PAM) and multiple $\mu$ LEDs (optically-generated PAM) are shown in Fig. 1(c). Multiple $\mu$ LEDs are able to generate more optical power and therefore, provide extra power budget compared with a single $\mu$ LED link. Moreover, the non-linear behaviour of the $\mu$ LEDs is mitigated resulting in a smaller nonlinearity penalty in the link. However, a larger extinction penalty is expected in the multiple $\mu$ LED
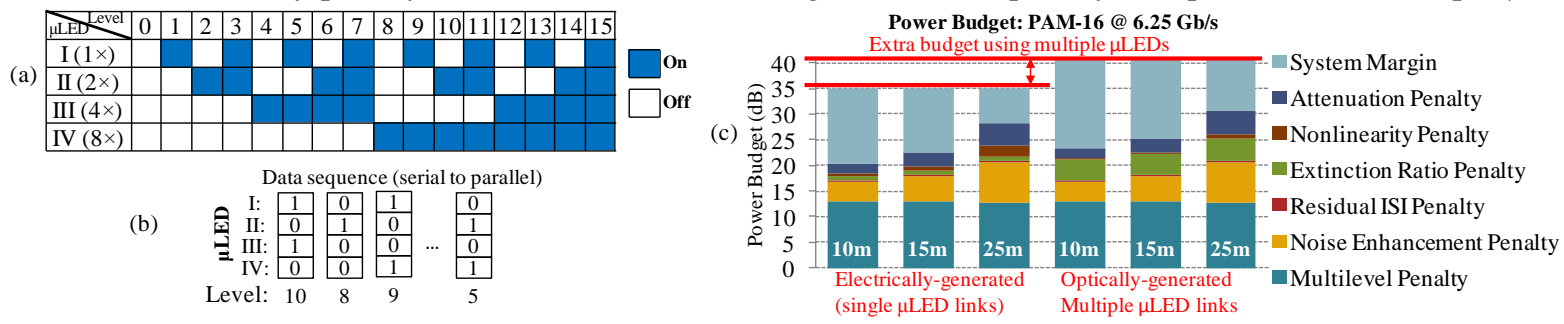

Fig. 1 Schematic of (a) PAM-16 generation using $4 \mu$ LEDs, (b) modulating data sequence for each $\mu$ LED and (c) power budget analysis results. 
links due to the required DC bias to all of the $\mu$ LEDs. The simulation results show that the multiple $\mu$ LED scheme offers a $3.8 \mathrm{~dB}$ greater link power margin for a PAM-16 modulation and $6.25 \mathrm{~Gb} / \mathrm{s}$ data rate for all POF lengths.

\section{Experimental setup and results}

The experimental setup used in the demonstration of the optically generated PAM POF link is illustrated in Fig. 2(a). Two $\mu$ LED-array boards, each operating with two $450 \mathrm{~nm} \mu \mathrm{LEDs}$, are used. The output beams of the $4 \mu$ LEDs are combined using a beam splitter and coupled into POF using aspherical lenses [Fig. 2(b)]. At the receiver end, an avalanche photodiode (APD) is employed to detect the transmitted signal and the resultant waveform is captured using a digital communication analyzer for offline processing. 4 independent data streams from a pulse pattern generator (PPG) are employed to drive the $4 \mu \mathrm{LEDs}$, while the operation (bias, RF amplitude) of each $\mu$ LED is adjusted so that the optical power received at the APD from each $\mu$ LED follows the 1:2:4:8 ratio. Finally, the electrical delays of the 4 input data streams are appropriately adjusted to synchronize all $4 \mu$ LEDs.

(a)

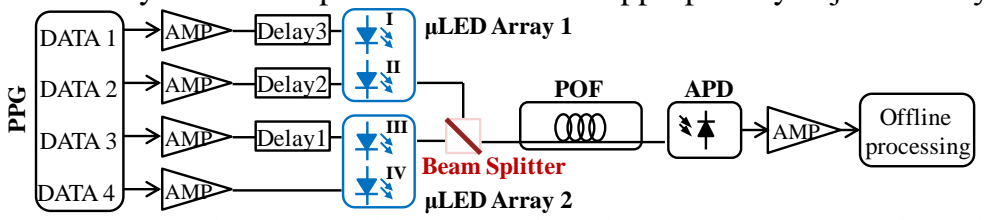

(b)

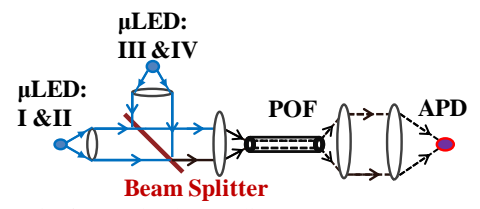

Fig. 2 (a) Experimental setup for optically implemented PAM link and (b) schematic for optical coupling

The received PAM-16 waveform at $6.25 \mathrm{~Gb} / \mathrm{s}$ is shown in Fig. 3(a) with the equivalent ideal PAM-16 signal noted. The waveform is severely distorted due to the link bandwidth limitation while the corresponding eye diagram is completely closed [Fig. 3(b)]. Offline processing consisting of a feed-forward equalizer (FFE) and a decisionfeedback equalizer (DFE) is used to retrieve the transmitted data sequence. The equalized eye-diagram has clearly distinguishable 16 levels [Fig. 3(c)]. The respective BER can be calculated using the equalized waveform and the measured APD noise performance. The BER performance of the link is evaluated at various symbol rates for the optical back-to-back case (B2B, short POF patchcord) and $10 \mathrm{~m}$ of POF [Fig. 3(d)]. The highest data rate achieved using the proposed scheme is $6.25 \mathrm{~Gb} / \mathrm{s}$. A BER $<10^{-3}$ is achieved after transmission over $10 \mathrm{~m}$ of POF. The penalty for the optical B2B link and $10 \mathrm{~m}$ POF link is measured to be $\sim 1 \mathrm{~dB}$.

(a)

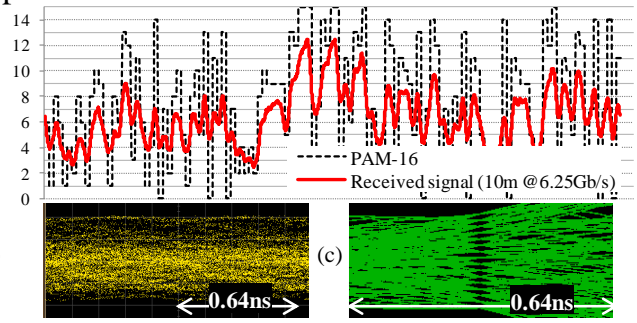

(d)

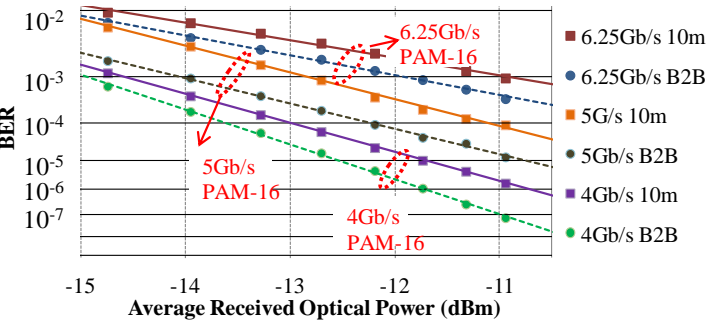

Fig. 3 (a) Experimentally captured waveform, (b) eye-diagram and (c) equalized eye-diagram for $6.25 \mathrm{~Gb} / \mathrm{s}$ PAM-16 link over $10 \mathrm{~m}$ of POF; (d) BER performance for optically implemented PAM-16 link at 4, 5 and $6.25 \mathrm{~Gb} / \mathrm{s}$ over b2b and $10 \mathrm{~m}$ of POF

\section{Conclusion}

In this work, we propose the use of an optically-generated PAM scheme using multiple $\mu$ LEDs in order to achieve a high data rate transmission over low-cost POF links. Simulation shows a $3.8 \mathrm{~dB}$ greater link power margin using the proposed technique and $6.25 \mathrm{~Gb} / \mathrm{s}$ data transmission over $10 \mathrm{~m}$ of POF using $4 \mu \mathrm{LEDs}$ and a PAM-16 modulation is experimentally demonstrated with a BER $<10^{-3}$.

5. Acknowledgements: This work is supported by the UK Engineering and Physical Sciences Research Council (EPSRC) via the Ultra Parallel Visible Light Communication (UP-VLC) Project.

\section{Reference}

[1] O. Ziemman, J. Krauser, P. E. Zamzow and W. Daum POF Handbook: Optical Short Range Transmission Systems, 2008 :Springer-Verlag

[2] X. Li et al., "Avalanche Photodiode Enhanced PAM-32 5 Gb/s LED-POF Link," Proc. ECOC, P.4.8, Cannes (2014).

[3] H. Elgala, R. Mesleh, H. Haas, "Indoor optical wireless communication: potential and state-of-the-art," Communications Magazine, IEEE , vol.49, no.9, pp.56-62, September 2011

[4] J. Armstrong, "Optical domain digital-to-analog converter for visible light communications using LED arrays" Photon. Res. 1, 92-95 (2013).

[5] T. Fath, C. Heller, and H. Haas, “Optical wireless transmitter employing discrete power level stepping," JLT. 31, 1734-1743 (2013).

[6] C. Xi; A. Mirvakili, V.J. Koomson, "A Visible Light Communication System Demonstration Based on 16-Level Pulse Amplitude Modulation of an LED Array," Photonics and Optoelectronics (SOPO), 2012 Symposium on , vol. 1, no. 4, pp. 21-23, May 2012

[7] M. Noshad, M. Brandt-Pearce, "Multilevel pulse-position modulation based on balanced incomplete block designs," Global Communications Conference (GLOBECOM), 2012 IEEE , pp.2930,2935, 3-7 Dec. 2012

[8] H. Roscher, et al. "Toward more efficient fabrication of high-density 2-D VCSEL arrays for spatial redundancy and/or multi-level signal communication." Photonics Europe. International Society for Optics and Photonics, 2006.

[9] J. J. D. McKendry, D. Massoubre, S. Zhang, B. R. Rae, R. P. Green, E. Gu, R. K. Henderson, A. E. Kelly, and M. D. Dawson, "Visible-Light Communications Using a CMOS-Controlled Micro-Light- Emitting-Diode Array,” J. Light. Technol., vol. 30, no. 1, pp. 61-67, Jan. 2012. 